\title{
BLAZHKO EFFECT IN THE RR LYRAE VARIABLES AS A RESULT OF DOUBLE MODE
}

PULSATION

\author{
K.J. Borkowski \\ Wrocław University Observatory, Wrocław, Poland
}

\section{ABSTRACT}

On the basis of a frequency analysis of light variations of AR Her a double-mode pulsation is proposed as an explanation of the Blazhko effect. The analysis revealed a number of components, which can all be interpreted as a result of nonlinear superposition of the fundamental mode, and a mode with period $P=1 /\left(2 / P_{0}+1 / P_{B}\right) \simeq P_{0} / 2$, where $P_{0}=0.470$ and $P_{B}=31.55$ denote the fundamental and the Blazhko effect periods, respectively.

The $P \simeq P_{0} / 2$ mode can be identified as the second or the third radial overtone. In both these cases the "beat" masses derived from a comparison of the calculated (nonadiabatic) and observed period ratios for RR Lyrae variables with Blazhko effect are different from their evolutionary masses.

\section{INTRODUCTION}

A number of RR Lyrae variables show so called Blazhko effect. It consists in periodic changes of the shape of light and radial velocity curves and also of the phases of 1 ight and velocity maxima. The Blazhko effect periods are typically in the range of $25^{\mathrm{d}}-100^{\mathrm{d}}$, i.e., about a hundred times the pulsation period. A summary of the observations of Blazhko effect has been recently provided by Szeidl (1976).

A number of hypotheses have been advanced in order to explain this effect, beginning with the hypothesis of Kluyver (1936) who assumed that in addition to the fundamental mode, which is the dominant one in RRab variables, a higher radial mode, with the period twice shorter than the fundamental, is also excited. None of them, however, was adequately supported by either observations or theory. This applies also to the most popular hypothesis, which invokes magnetic field in a rotating star and identifies the Blazhko and rotational periods (Detre and Szeidl 1973).

In this situation it seemed worthwhile to investigate frequency spectra of RR Lyrae stars which show Blazhko effect. Therefore the author per-

Space Science Reviews 27 (1980) 511-517. 0038-6308/80/0274-511\$01.05.

Copyright $\odot 1980$ by D. Reidel Publishing Co., Dordrecht, Holland, and Boston, U.S.A. 
formed a frequency analysis of the light variations of AR Her, one of the well-known stars of this type. The results (Borkowski 1980a) are briefly discussed in the present paper. It is pointed out that Blazhko effect should be interpreted as resulting from simultaneous excitation of two normal pulsation modes. Furthermore, it is shown that interpretation in terms of double mode radial pulsation leads to a mass problem.

\section{DOUBLE MODE PULSATION AS AN EXPLANATION OF THE BLAZHKO EFFECT}

The fundamental period of $A R H e r$ is equal to $P_{0}=0.470$, and the Blazhko effect period amounts to $P_{B}=31.55$, which is rather short. As data for the analysis a series of 1363 visual observations obtained by Tsessevich. on 114 nights in 1944 (Tsessevich and Ustinov 1953) were selected. The time interval between the first and last observation amounted to about $210^{d}$.

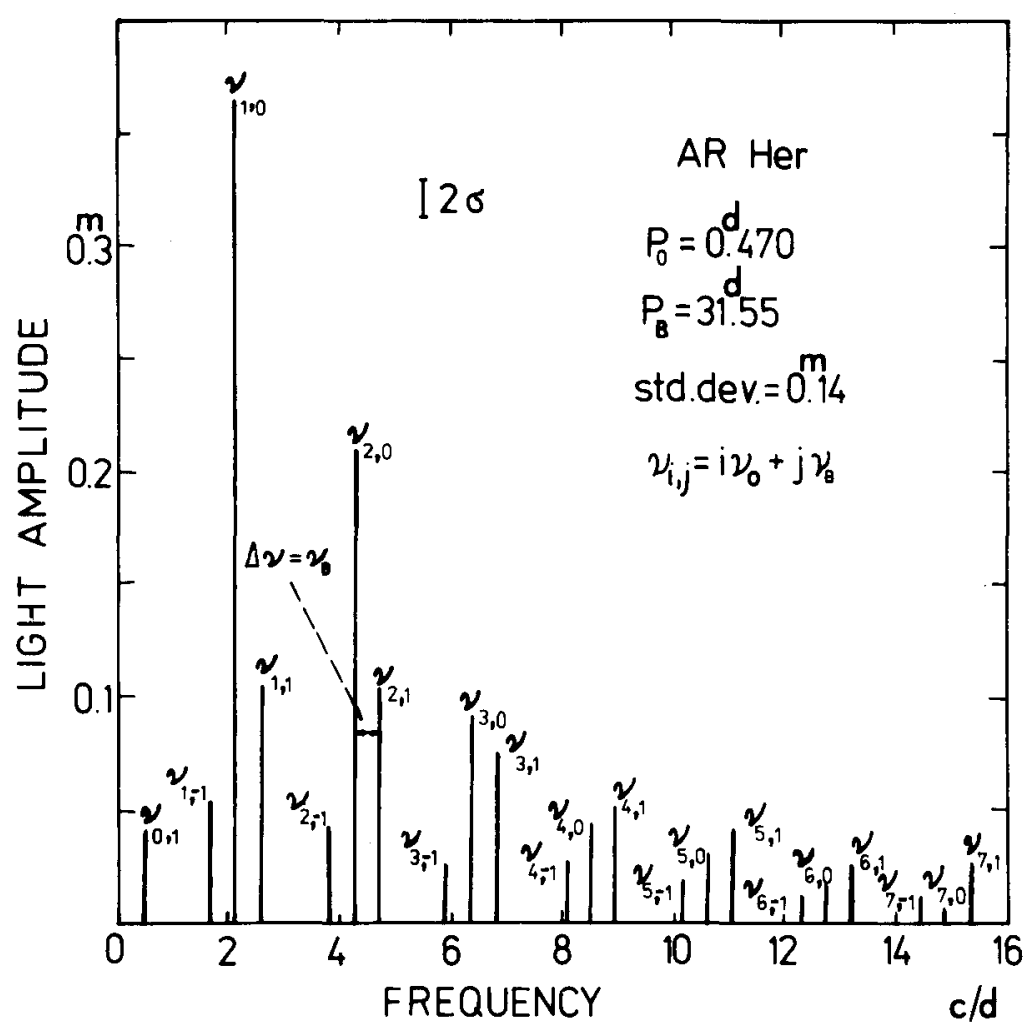

Figure 1. Frequency spectrum of AR Her. Vertical bar corresponds to twice the mean square error of the amplitudes. In order to avoid crowding, lines corresponding to closely spaced frequencies like $2 v_{0}$ and $2 \nu_{0}+\nu_{B}$ are plotted with the separations increased about 15 times. 
Frequency analysis of Tsessevich's data revealed a number of components hidden in the light variation. Frequencies of all components turned out to be of the form $i v_{0}+j \nu_{B}$, where $\nu_{0}=1 / P_{0}$ and $\nu_{B}=1 / P_{B}$ denote the fundamental and the $B$ lazhko effect frequeltcies, respectively. The component light amplitudes are plotted as a function of frequency in Fig. 1. The solution corresponding to the frequency spectrum shown in Fig. 1 fits the data fairly well: the standard deviation amounts to 0.14 which is only slightly larger than the accuracy of a single visual observation.

The frequency spectrum of AR Her can be most naturally explained as follows (see Fig.2). Two pulsation modes, with the periods in the ratio very close to $1: 2$, are simultaneously excited. AR Her pulsates in the fundamental mode the period of which is equal to 0.470 . Strong excitation of this mode explains why components with frequencies $v_{0}, 2 v_{0}$, $3 v_{0}$, etc., are present in the frequency spectrum. The eigenfrequency of the second mode is slightly larger than $2 v_{0}$ viz., it is equal to $2 v_{0}+v_{B}$, and the corresponding period is 8.233 . Due to the $2: 1$ commensurability the two modes excited in AR Her are in resonance. This interpretation is reminiscent of the above-mentioned hypothesis of Kluyver (1936).

As in the case of double mode pulsators nonlinear superposition of the pulsation modes is seen in AR Her. The first order nonlinear superposition terms, with frequencies equal to $v-v_{0}$ and $v+v_{\rho}$, the second order $2 v_{0}+v$ and $v-2 v_{0}$ terms, and many other of higher order are present in the frequency spectrum. It is possible to interpret all components found in the light variation of AR Her as resulting from nonlinear superposition of the pulsation modes. Consistent with this interpretation of the Blazhko effect is the fact that higher order components have, as a rule, lower amplitudes. However, due to resonant effects the exact relation between amplitudes and the order of nonlinear superposition does not hold. In particular, the resonant components whose frequencies are very close to the strongly excited fundamental, i.e., the $v-v_{0}$ and $3 v_{0}-v$ terms, have slightly larger amplitudes than one would expect from their order.

The small value of the frequency $\nu_{B}=\nu-2 \nu_{0}$, strong nonlinear mode superposition, and resonance effects make the beat phenomenon caused by the simultaneous excitation of the two pulsation modes the most conspicuos feature of the light variation of AR Her. This explains why the Blazhko effect has been so of ten considered as modulation with the beat frequency equal to $v_{B}$.

\section{THE MASS DISCREPANCY}

A question arises what is the pulsation mode, radial or nonradial, which corresponds to the $v=2 v_{0}+v_{B}$ component. It can be assumed, as the simplest possibility, that a radial overtone is involved. In order to find out which of the radial overtones would be the most appropriate one, nonadiabatic periods were calculated for the fundamental mode, second overtone and third overtone using RR Lyrae models in the center 


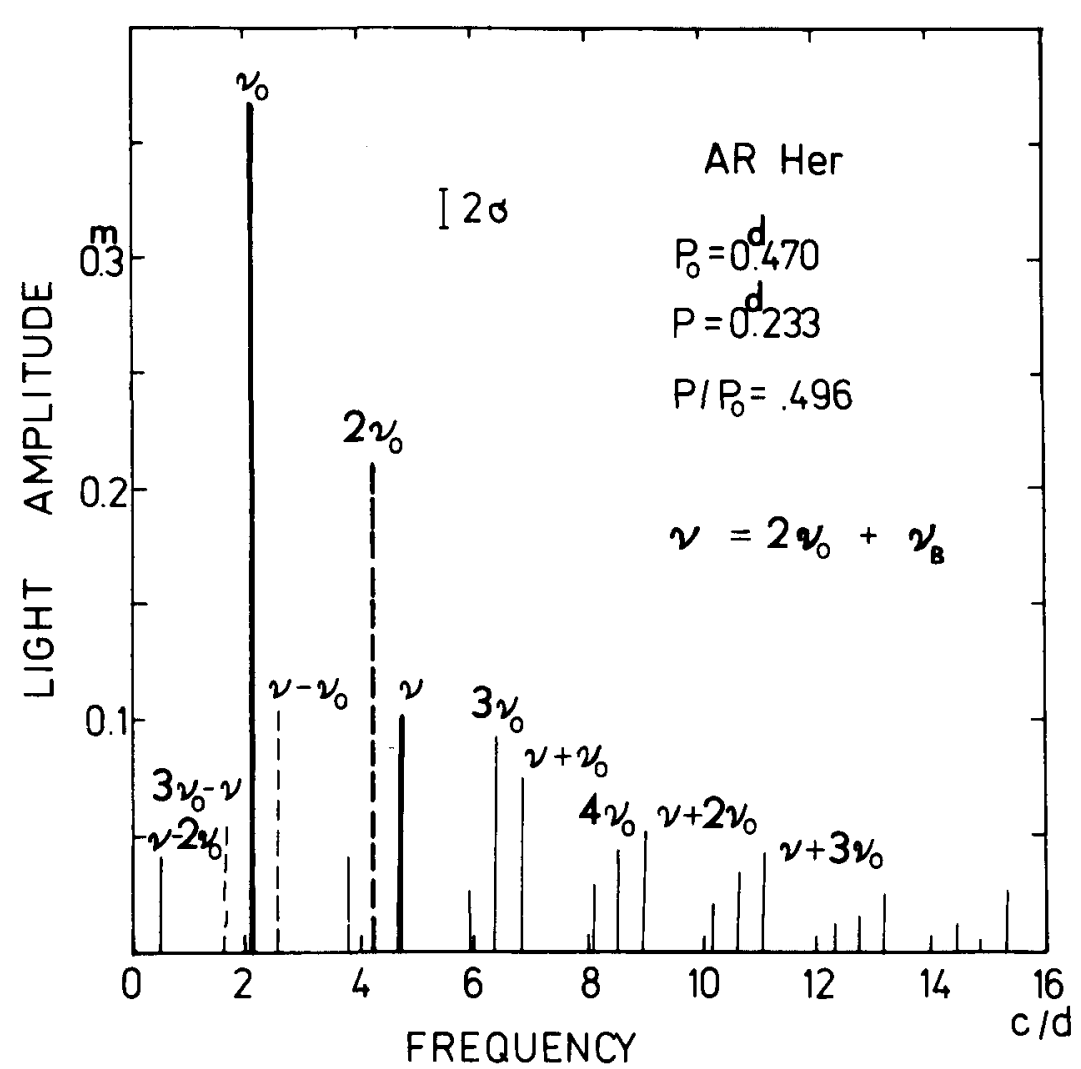

Figure 2. Interpretation of the frequency spectrum of AR Her. Thick solid lines denote excited modes while thin dashed lines indicate resonant components. The important $2 v_{0}$ component is drawn by thick dashed line. Vertical bar corresponds to twice the mean square error of the amplitudes.

of the instability strip. They had the standard population 11 composition $X=0.7$ and $Z=0.001$. The results are shown in Fig. 3 where the period ratio is plotted as a function of logarithm of the fundamental period.

Together with the second and the third overtone theoretical lines of constant mass the observational data (circles) are also shown. The periods were taken from Szeidl (1976) for 26 field variables, and from Goranskij (1976) and Jørgensen (1980) for four globular cluster variables. However, except for the Jørgensen's variables, only the Blazhko effect periods are known for the other stars instead of the $\nu$ mode periods. This gives $r i s e$ to an ambiguity in the period of the $v$ mode since two its values, viz., $1 /\left(2 / P_{0}+1 / P_{B}\right)$ and $1 /\left(2 / P_{0}-1 / P_{B}\right)$, are possible. Nevertheless, the author (Borkowski ${ }^{B} 1980$ b) has been able to determine the correct periods for some variables using observed phase relations between the times and heights of the light maxima. Independently, the $v$ 


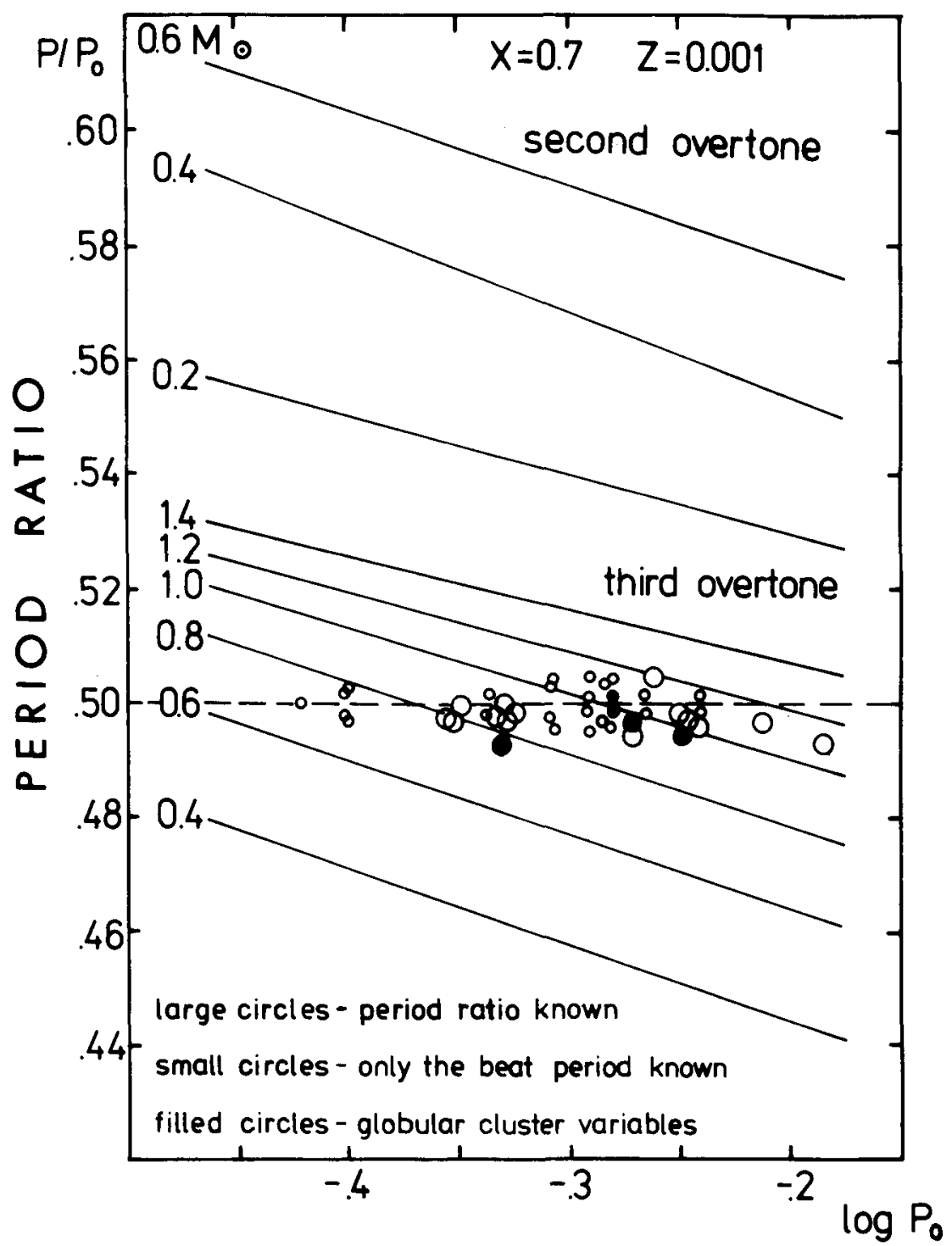

Figure 3. Theoretical ( lines) and observed (circles) period ratios of RR Lyrae stars.

mode periods of two of these variables were found by Wal raven (1955) for RR Lyr and the author (Borkowski 1980a, see also Chapter 2) for AR Her by means of detailed analyses of their light variations. In Fig.3 variables for which the period of the $v$ mode is known are shown with large circles while small circles indicate those stars for which only the Blazhko effect period has been determined. In the latter case two small circles corresponding to two possible periods of the $v$ mode are plotted for each variable. Filled circles denote globular cluster variables. 
A comparison of the observed and theoretical period ratios makes it possible to determine masses of the RR Lyrae stars with Blazhko effect. As can be seen from Fig. 3 these so called "beat" masses are lower than $0.2 \mathrm{M}$ if the second overtone is identified with the $\nu$ mode, or are in the range from 0.7 to at least $1.1 \mathrm{M}$ if the third overtone is as sumed. In both these cases they differ from ${ }^{\circ}$ the $0.5-0.7 \mathrm{M}$ evolutionary masses of the RR Lyrae variables. The "beat" masses are unre̊alistically small for the second overtone hypothesis, and slightly too large if the third overtone were assumed. As is well known, similar discrepancy occurs al so for the double mode and bump Cepheids, viz., their evolutionary masses are much larger than those derived by means of pulsation theory ( $c f$. review articles by Cox (1980) and Stobie (1980)). The mass problem is thus common to both Cepheids and RR Lyrae variables with Blazhko effect. Because of the mass discrepancy it cannot be definitely decided at present whether the second or the third overtone is excited in variables with Blazhko effect. However, both Cepheid and RR Lyrae mass problems are identical, i.e., masses derived by means of pulsation theory are lower than the evolutionary ones, only when the second overtone hypothesis is assumed. This suggests that the second overtone should be identified with the $v$ mode.

Undertaking the above research was suggested by $\operatorname{Dr}$. M.Jerzykiewicz which I gratefully acknowledge. I am also indebted to Dr. W.Dziembowski for supplying the models and pulsation programme and for many invaluable discussions. Finally, I wish to thank Dr. H.E.Jørgensen who provided me with his unpublished results.

\section{REFERENCES}

Borkowski, K.J.:1980a, Acta Astronomica 30, in press.

Borkowski, K.J.:1980b, in preparation.

Cox, J.P.: 1980, this proceedings.

Detre, L., Szeidl, B.: 1973, "Variable Stars in Globular Clusters and in Related Systems", ed. J.D.Fernie,pp.31-34.

Goranskij, V.P.:1976, Variable Stars Suppl. 2,pp.323-330.

Jørgensen, H.E.:1980, private communication.

Kluyver, M.: 1936, B.A.N. 7,pp.313-323.

Stobie, R.S.: 1980 , this proceedings.

Szeidl, B.:1976, "Multiple Periodic Variable Stars",ed. W.S.Fitch, Part 1,pp.133-151.

Tsessevich, V.P., Ustinov, B.A.:1953, Trudy Gos.Astr. Inst.Sternberga 23, pp. 62-249.

Walraven, Th.:1955, B.A.N. 12, pp. 223-265. 


\section{DISCUSSION}

SIMON: I'm happy to hear about this work. I don't like it that the RR Lyrae stars are so well understood. How good do you match the light curve for these stars executing the Blashko effect?

JERZYKIEWICZ: All we can do is to analyze just one season's observations. This is the main problem, because the Blashko effect is not constant. It changes from one season to another. It may be related somehow to the interior structure, semiconvection, or something like that. Period changes are very common in RR Lyrae stars and Iong term period changes complicate the Blashko effect.

SIMON: Did you pick the light curve at a given epoch?

JERZYKIEWICZ: Borkowski used only one season's observations, hoping the Blashko effect would not change in that one season. There must be some other phenomena going on which make it impossible to do a periodogram analysis from a very long data set.

FITCH: Didn't Walraven have some data?

JERZYKIEWICZ: I don't think so. You need data from a single season, say 30 nights, and that is difficult to find. We plan to observe these stars, but it is, you know, a long process.

PERCY: What fraction of all the RR Lyrae stars show this effect?

FITCH: My guess, not having looked up the numbers, is the order of 1/3. Maybe that's too high.

JERZYKIEWICZ: Maybe 20\%.

PERCY: It seems that too many stars show such an exact coincidence or resonance. If all the stars have roughly the same mass, you would expect the Blashko effect to occur at roughly the same period. There would be an intersection at only one point in your diagram.

JERZYKIEWICZ: Why should all the masses be exactly the same?

PERCY: I guess they don't have to be, but the range is rather large. JERZYKIEWICZ: Yes. That's true.

COGAN: If you look at theoretical models the effective mean polytropic index primarily determines the period ratio. Even if these stars have a modest range in mass $\mathrm{T}_{e}$, and luminosity they are basically all the same in terms of envelope structure. Period ratios will always be quite close together.

JERZYKIEWICZ: We have used only one composition, population II. These stars have a range of composition. Does that influence the period ratio?

COGAN: Very little. 